\title{
Incorporating Feedforward Action into Self-optimizing Control Policies
}

\author{
Lia Maisarah Umar ${ }^{\dagger}, \mathrm{Yi} \mathrm{Cao}^{\ddagger}$ and Vinay Kariwala ${ }^{\S *}$ \\ $\dagger$ School of Chemical and Biomedical Engineering, \\ Nanyang Technological University, Singapore 637459 \\ ¥ School of Engineering, Cranfield University, Cranfield, Bedford MK43 0AL, UK \\ $\S$ ABB Global Industries \& Services Ltd, Mahadevpura, Bangalore 560048, India
}

This version: November 20, 2012

\begin{abstract}
The available methods for selection of controlled variables (CVs) using the concept of self-optimizing control (SOC) focus on finding CVs, which are held at constant setpoints using a feedback controller. In this paper, it is shown that better self-optimizing properties can be achieved by incorporating feedforward action using measured disturbances in the SOC policies. The resulting operational policy allows the setpoints for CVs to be varied with measured disturbances allowing for near-optimal operation in presence of disturbances and uncertainties. The effectiveness of the proposed approach is demonstrated through its application to an exothermic reactor and a forced-circulation evaporator.
\end{abstract}

Keywords: Controlled variable; Control structure design; Feedforward control; Self-optimizing control.

\section{Introduction}

The selection of controlled variables $(\mathrm{CVs})$ from available measurements is an important step during the design of control systems for industrial processes. A number of methods for CV selection have been proposed in the past few decades. Van de Wal and de Jager [2001] provide an overview of the CV selection approaches proposed in 1980s and 1990s. Recently, Skogestad [2000] introduced the concept of self-optimizing control (SOC) for systematic selection of CVs. In this approach, CVs are selected such that

\footnotetext{
${ }^{*}$ Corresponding author: E-mail: vinay.kariwala@in.abb.com
} 
in presence of disturbances and uncertainties, the loss incurred in implementing the operational policy by holding the selected CVs at constant setpoints is minimal, as compared to the use of an online optimizer.

The choice of CVs based on the general non-linear formulation of SOC requires solving large-dimensional non-convex optimization problems [Skogestad, 2000]. To quickly pre-screen alternatives, local methods have been proposed including the minimum singular value (MSV) rule [Skogestad and Postlethwaite, 1996] and exact local methods with worst-case [Halvorsen et al., 2003] and average loss minimization [Kariwala et al., 2008]. In comparison with the traditional approach of using a subset of available measurements as CVs, it is possible to obtain lower losses using linear combinations of available measurements as CVs [Halvorsen et al., 2003]. Recently, explicit solutions to the problem of finding locally optimal measurement combinations have been proposed [Alstad et al., 2009; Heldt, 2010; Kariwala, 2007; Kariwala et al., 2008]. The usefulness of these methods has been demonstrated through a number of case studies; see e.g. Rangaiah and Kariwala [2012] for an overview. Note that although the available methods can be used to evaluate the loss for the specified nonlinear combinations of measurements (e.g. ratios of measurements), these methods cannot find the optimal nonlinear measurement combination that minimizes loss. Jaschke and Skogestad [2012] have provided a partial solution to overcome this problem, which allows using polynomials expressed in terms of measurements as CVs, but the effect of measurement noise is ignored.

The past development in SOC has focussed on the selection of CVs, which are held at constant setpoints using feedback controller to achieve near-optimal operation. In practice, measurements of some key disturbances are often available. These disturbances can include the physical disturbances, which affect the process outputs, as well as "virtual" disturbances, which do not affect the outputs, but have an effect on economics, e.g. utility cost. Clearly, the loss incurred in implementing the SOC policy can be reduced by adding feedforward action based on these measured disturbances to the feedback controller. This possibility has been earlier mentioned by Alstad [2005], but no analysis was presented. Cao and Yang [2004] considered the use of measured virtual disturbances to enhance the SOC policies under the restrictive assumption that these disturbances are measured perfectly.

In this paper, we present an exact local method for SOC, which allows incorporating feedforward action in SOC policies using noisy measurements of both physical and virtual disturbances. In particular, it is shown that loss can be reduced by including the measured disturbances in the available measurement set (typically consisting of outputs and inputs) and finding locally optimal measurement combinations of the extended measurement set. The resulting control system can be viewed as one, where the feedback controller holds the CVs at setpoints, which vary with the disturbances [Cao and Yang, 2004]. The effectiveness of the 
proposed approach is demonstrated through its application to an exothermic reactor [Economou et al., 1986; Kariwala, 2007] and a forced-circulation evaporator [Kariwala et al., 2008; Newell and Lee, 1989].

The rest of the paper is organized as follows: the available local methods for SOC are briefly discussed in Section 2. The proposed approach of incorporating feedforward action into SOC policies is presented in Section 3. Case studies on the exothermic reactor and forced-circulation evaporator are given in Section 4 to illustrate the advantages of the proposed approach. Finally, Section 5 concludes the paper.

\section{Local Methods for Self-Optimizing Control}

Consider that the steady-state economics of the plant is characterized by the scalar objective function $J(u, d)$, where $u \in \mathbb{R}^{n_{u}}$ and $d \in \mathbb{R}^{n_{d}}$ are inputs and disturbances, respectively. Let the linearized model of the process, obtained around the nominally optimal operating point, be

$$
y=G^{y} u+G_{d}^{y} W_{d} d+W_{e} e
$$

where $y \in \mathbb{R}^{n_{y}}$ denotes the measurements of process variables (PVs) and $e \in \mathbb{R}^{n_{y}}$ denotes the implementation error, which results due to measurement and control errors. Here, the diagonal matrices $W_{d}$ and $W_{e}$ contain the expected magnitudes of disturbances and implementation error, respectively. The CVs $c \in \mathbb{R}^{n_{u}}$ are given as

$$
c=H y=G u+G_{d} W_{d} d+H W_{e} e
$$

where $H$ is a selection or combination matrix and

$$
G=H G^{y}, \quad G_{d}=H G_{d}^{y}
$$

It is assumed that $G \in \mathbb{R}^{n_{u} \times n_{u}}$ is invertible. This assumption is necessary for integral control. When $d$ and $e$ are assumed to be uniformly distributed over the set

$$
\left\|\left[\begin{array}{ll}
d^{\prime} & e^{\prime}
\end{array}\right]^{\prime}\right\|_{2} \leq 1
$$

where "'" represent the transpose of a matrix, the local worst-case and average losses are given as [Halvorsen et al., 2003; Kariwala et al., 2008]:

$$
\begin{aligned}
L_{\text {worst }}(H) & =0.5 \bar{\sigma}^{2}\left(J_{u u}^{1 / 2}\left(H G^{y}\right)^{-1} H Y\right) \\
L_{\text {average }}(H) & =\frac{1}{6\left(n_{y}+n_{d}\right)}\left\|J_{u u}^{1 / 2}\left(H G^{y}\right)^{-1} H Y\right\|_{F}^{2}
\end{aligned}
$$


where $n_{y}$ and $n_{d}$ represent the number of PVs and disturbances, respectively, $\bar{\sigma}$ and $\|\cdot\|_{F}$ denote the maximum singular value and Frobenius norm, respectively, and

$$
Y=\left[\left(G^{y} J_{u u}^{-1} J_{u d}-G_{d}^{y}\right) W_{d} W_{e}\right]
$$

with $J_{u u}=\frac{\partial^{2} J}{\partial u^{2}}$ and $J_{u d}=\frac{\partial^{2} J}{\partial u \partial d}$, evaluated at the nominal operating point. In comparison with worst-case loss, the selection of CVs is preferred through minimization of average loss, as the worst-case may not occur frequently in practice [Kariwala et al., 2008].

When individual measurements are selected as CVs, $H$ can be considered to be a selection matrix. The selection of $n_{u} \mathrm{CVs}$ from $n_{y}$ measurements is a combinatorial optimization problem. Bidirectional branch and bound (BAB) methods have been proposed in [Kariwala and Cao, 2009, 2010] to solve this problem efficiently.

Instead of using individual measurements, it is possible to use combinations of measurements as CVs. For this case, Alstad et al. [2009] has recently proposed an explicit expression for $H$, which minimizes the $L_{\text {average }}$ in $(6)$ and is given as

$$
H^{\prime}=\left(Y Y^{\prime}\right)^{-1} G^{y}\left(\left(G^{y}\right)^{\prime}\left(Y Y^{\prime}\right)^{-1} G^{y}\right)^{-1} J_{u u}^{1 / 2}
$$

As shown by Kariwala et al. [2008], the $H$ in (8) also minimizes $L_{\text {worst }}$ in (5). The locally optimal combinations of all the available measurements, which can be used as CVs can be found using (8). It is, however, noted in [Alstad et al., 2009; Kariwala, 2007; Kariwala et al., 2008] that the use of combinations of a few measurements as CVs often provide similar loss as the case where combinations of all available measurements are used. Partially bidirectional BAB methods have been proposed for efficient selection of measurements, whose combinations can be used as CVs, in [Kariwala and Cao, 2009, 2010].

Remark 1: For the given locally optimal combination matrix $H$, any combination matrix obtained as $Q H$, where $Q$ is a non-singular matrix, is also locally optimal [Halvorsen et al., 2003]. Thus, by selecting $Q=\left(\left(G^{y}\right)^{\prime}\left(Y Y^{\prime}\right)^{-1} G^{y}\right) J_{u u}^{-1 / 2}$, a simplified expression for locally optimal $H$ can be derived as [Rangaiah and Kariwala, 2012]

$$
H^{\prime}=\left(Y Y^{\prime}\right)^{-1} G^{y}
$$

To find $Y$ in (7), one needs to evaluate $\left(G^{y} J_{u u}^{-1} J_{u d}-G_{d}^{y}\right)$, which represents the sensitivity of the optimal values of $y$ with respect to $d$ and can be obtained by repeatedly solving the non-linear optimization problem with perturbations in $d$ [Alstad et al., 2009]. Then, (9) shows that to find locally optimal $H$, the knowledge of $J_{u u}$ is not necessary, which can be difficult numerically, especially if the process is modelled using a 
commercial simulator; see e.g. [Yelchuru and Skogestad, 2012]. However, $J_{u u}$ is still required to evaluate loss, e.g. during the application of $\mathrm{BAB}$ methods for measurement subset selection.

\section{Incorporation of disturbance measurements into SOC}

To incorporate feedforward action into SOC policies, we partition $d$ into $d_{m}$ (measured disturbances) and $d_{u}$ (unmeasured disturbances). The disturbance gain matrix is conformably partitioned as

$$
G_{d}^{y}=\left[\begin{array}{ll}
G_{d_{m}}^{y} & G_{d_{u}}^{y}
\end{array}\right]
$$

The measured value of $d_{m}$ is denoted as $\hat{d}_{m}$. It is considered that the elements of the diagonal matrix $W_{e_{d}}$ contain the error associated with measurement of $d_{m}$. Let us define the extended measurement set as

$$
\tilde{y}=\left[\begin{array}{ll}
y^{\prime} & \hat{d}_{m}^{\prime}
\end{array}\right]^{\prime}
$$

It follows that

$$
\tilde{y}=G^{\tilde{y}} u+G_{d}^{\tilde{y}} W_{d} d+W_{\tilde{e}} \tilde{e}
$$

where

$$
\begin{aligned}
G^{\tilde{y}} & =\left[\begin{array}{c}
G^{y} \\
0
\end{array}\right] \\
G_{d}^{\tilde{y}} & =\left[\begin{array}{cc}
G_{d_{m}}^{y} & G_{d_{u}}^{y} \\
I & 0
\end{array}\right] \\
W_{\tilde{e}} & =\left[\begin{array}{cc}
W_{e} & 0 \\
0 & W_{e_{d}}
\end{array}\right] \\
\tilde{e} & =\left[\begin{array}{ll}
e^{\prime} & e_{e_{d}}^{\prime}
\end{array}\right]^{\prime}
\end{aligned}
$$

with $e_{e_{d}}$ satisfying $W_{e_{d}} e_{e_{d}}=\hat{d}_{m}-d_{m}$.

Based on the extended set of measurements, the CVs can be selected as

$$
\tilde{c}=\tilde{H} \tilde{y}=H_{y} y+H_{d_{m}} \hat{d}_{m}
$$

Similar to (8), the optimal $H$ can be obtained using the following expression

$$
\tilde{H}^{\prime}=\left(\tilde{Y} \tilde{Y}^{\prime}\right)^{-1} G^{\tilde{y}}\left(\left(G^{\tilde{y}}\right)^{\prime}\left(\tilde{Y} \tilde{Y}^{\prime}\right)^{-1} G^{\tilde{y}}\right)^{-1} J_{u u}^{1 / 2}
$$


where

$$
\begin{aligned}
& \tilde{Y}=\left[\left(G^{\tilde{y}} J_{u u}^{-1} J_{u d}-G_{d}^{\tilde{y}}\right) W_{d} \quad W_{\tilde{e}}\right] \\
& =\left[\begin{array}{ccc}
\left(G^{y} J_{u u}^{-1} J_{u d}-G_{d}^{y}\right) W_{d} & W_{e} & 0 \\
-W_{d_{m}} & 0 & W_{e_{d}}
\end{array}\right]
\end{aligned}
$$

Here, the diagonal matrix $W_{d_{m}}$ contains the magnitude of $d_{m}$. In the proposed approach, $u$ is manipulated such that $\tilde{c}=\tilde{H} \tilde{y}=H_{y} y+H_{d_{m}} \hat{d}_{m}=0$ or

$$
H_{y} y=-H_{d_{m}} \hat{d}_{m}
$$

Interpreting $H_{y} y$ as the $\mathrm{CV}$ for feedback control and $-H_{d_{m}} \hat{d}_{m}$ as its setpoint, the proposed operational policy is viewed as traditional SOC approach with varying setpoint. Note that in comparison with feedback only, the loss seen using the feedforward action cannot be higher, as in the worst-case $H_{d_{m}}$ can always be selected to be zero and $H_{y}$ as $H$ in (8).

\section{Results and Discussion}

In this section, we demonstrate the advantages of incorporating feedforward action into SOC policies using benchmark examples.

\subsection{Exothermic Reactor}

We first consider the example of an exothermic reactor [Economou et al., 1986] to illustrate the benefit of incorporating measured physical disturbances into the measurement set. For this process, the inlet to the well-mixed reactor consists of reactant $A$ and product $B$ as contaminant at temperature $T_{i}$. The outlet stream has a temperature $T$ with the concentrations of the product $B$ and the unused reactant $A$ being $C_{B}$ and $C_{A}$, respectively. The schematic of the exothermic reactor is shown in Figure 1. The economic objective function to be minimized is

$$
J=-2.009 C_{B}+\left(0.001657 T_{i}\right)^{2}
$$

This process has three outputs $\left(C_{A}, C_{B}\right.$, and $\left.T\right)$, one input $\left(T_{i}\right)$, and two disturbances $\left(C_{A i}\right.$ and $\left.C_{B i}\right)$. The nominal values of disturbances are $C_{A i}=1 \mathrm{~mol} / \mathrm{L}$ and $C_{B i}=0$. The measurement set is given as

$$
y=\left[\begin{array}{llll}
C_{A} & C_{B} & T & T_{i}
\end{array}\right]^{\prime}
$$




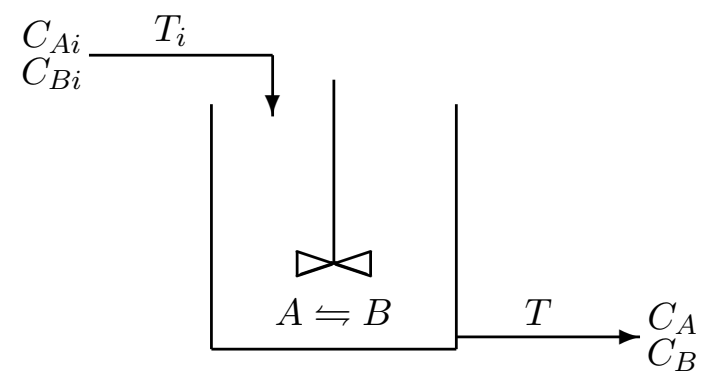

Figure 1: Schematic of Exothermic reactor

The allowable ranges for disturbances are $0.7 \leq C_{A i} \leq 1.3$ and $0 \leq C_{B i} \leq 0.3$, which implies that $W_{d}=\operatorname{diag}(0.3,0.3)$. The implementation errors are taken as $0.01 \mathrm{~mol} / \mathrm{L}$ for concentration measurements and $0.5 \mathrm{~K}$ for temperature measurement. Thus, $W_{e}=\operatorname{diag}(0.01,0.01,0.5,0.5)$. The gain and Hessian matrices obtained around the nominally optimal operating point are available in [Kariwala, 2007]. Let us define the extended measurement as $\tilde{y}=\left[\begin{array}{ll}y^{\prime} & d_{m}^{\prime}\end{array}\right]^{\prime}$. We consider four different cases, where $d_{m}=\emptyset$, $d_{m}=C_{A i}, d_{m}=C_{B i}$, and $d_{m}=\left[C_{A i} C_{B i}\right]^{\prime}$. Measurement errors for both disturbances $C_{A i}$ and $C_{B i}$ are taken as $0.01 \mathrm{~mol} / \mathrm{L}$.

The locally optimal measurement combination matrices for different measurement sets are computed using (18). The resulting losses for each CV candidate obtained using the local method and the nonlinear model are shown in Table 1. For nonlinear analysis, 100 scenarios are considered, where $d$ and $e$ are generated randomly to have zero mean and uniform distribution over the range -1 to 1 . In general, the nonlinear analysis shows good agreement with the local analysis, except that the worst-case loss seen with $d_{m}=\left[C_{A i} C_{B i}\right]^{\prime}$ is higher than the case with $d_{m}=C_{B i}$ for nonlinear analysis. This is due to the fact that the combination matrix $\tilde{H}$ obtained using (18) is only locally optimal. It can be noted that as compared to the use of feedback-based SOC policy, the inclusion of $C_{A i}$ and $C_{B i}$ individually reduces the average loss computed using nonlinear model by approximately $51 \%$, while inclusion of both disturbances results in $55 \%$ reduction.

Table 1: Local and nonlinear losses for exothermic reactor

\begin{tabular}{cccccc}
\hline \multirow{2}{*}{$d_{m}$} & \multicolumn{2}{c}{ Local Analysis } & & \multicolumn{2}{c}{ Nonlinear Analysis } \\
\cline { 2 - 3 } \cline { 5 - 6 } & $L_{\text {average }}[\$ / \mathrm{min}]$ & $L_{\text {worst }}[\$ / \mathrm{min}]$ & & Average loss $[\$ / \mathrm{min}]$ & Worst-case loss $[\$ / \mathrm{min}]$ \\
\hline$\emptyset$ & $1.465 \times 10^{-5}$ & $2.637 \times 10^{-4}$ & & $2.567 \times 10^{-4}$ & $2.372 \times 10^{-3}$ \\
$C_{A i}$ & $2.809 \times 10^{-6}$ & $5.899 \times 10^{-5}$ & & $1.256 \times 10^{-4}$ & $9.468 \times 10^{-4}$ \\
$C_{B i}$ & $1.856 \times 10^{-6}$ & $3.898 \times 10^{-5}$ & & $1.254 \times 10^{-4}$ & $6.699 \times 10^{-4}$ \\
$C_{A i}, C_{B i}$ & $1.182 \times 10^{-6}$ & $2.837 \times 10^{-5}$ & & $1.166 \times 10^{-4}$ & $9.304 \times 10^{-4}$ \\
\hline
\end{tabular}


Table 1 shows that the measurement set with $d_{m}=C_{B i}$ provides similar loss as seen with both disturbances measured. As combining fewer measurements is desirable to obtain a simpler control structure, we recommend the use of $C_{B i}$ as measured disturbance to incorporate feedforward action for this exothermic reactor. The selected $\mathrm{CV}$ is given as

$$
\tilde{c}=0.295 C_{A}-0.028 C_{B}-0.007 T-0.008 T_{i}-0.642 C_{B i}
$$

for which the relative average loss evaluated based on the nonlinear model is approximately $0.02 \%$ showing that the selected CV achieves nearly perfect self-optimizing properties.

(a)

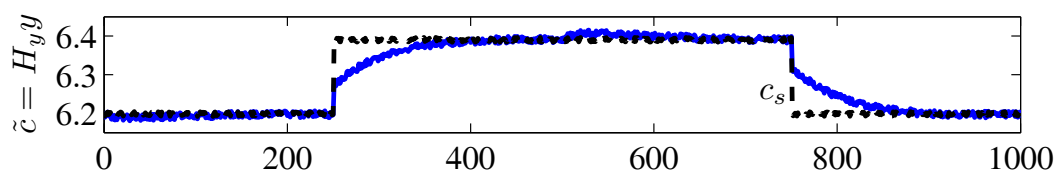

(b)

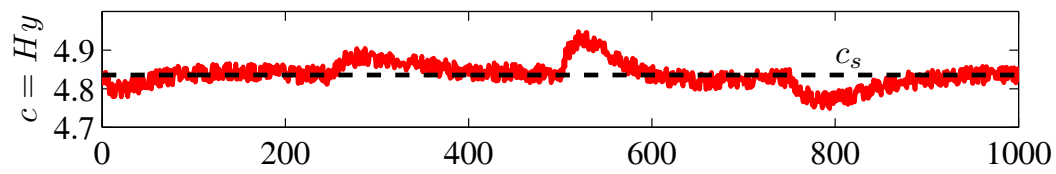

(c)

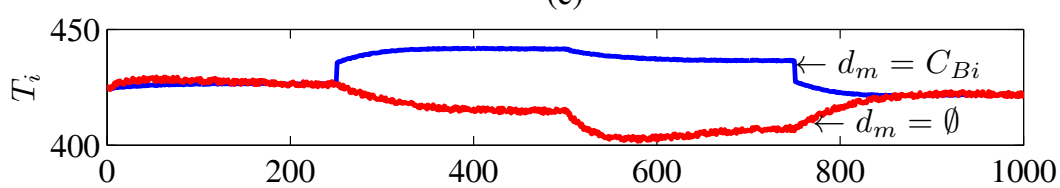

(d)

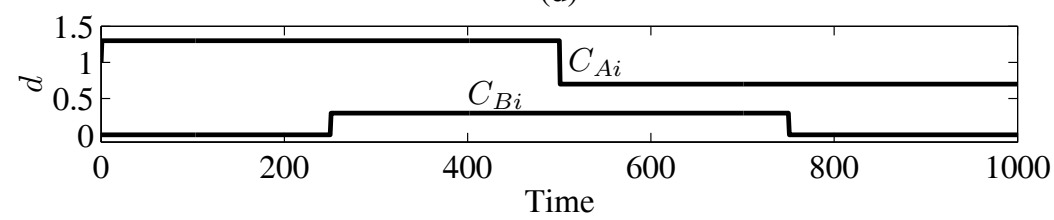

Figure 2: Comparison of dynamic responses for exothermic reactor: (a) $\mathrm{CV}\left(\tilde{c}=H_{y} y\right.$ ) for $d_{m}=C_{B i}$; (b) $\mathrm{CV}(c=H y)$ for $d_{m}=\emptyset ;(\mathrm{c})$ input $\left(T_{i}\right)$; and $(\mathrm{d})$ disturbances $(d)$

We point out that the proposed approach of selecting CVs as combinations of measurements does not take dynamic performance into account and the selected CVs can have poor controllability in general. To verify the dynamic performance, the following linear model, obtained around nominal optimal point, is derived:

$$
\frac{\tilde{c}(s)}{T_{i}(s)}=\frac{0.0159(30.964 s+1)(13.627 s+1)}{(60 s+1)(13.353 s+1)}
$$


A first order approximation of the model is obtained using half-rule and the internal model control (IMC) method [Skogestad, 2003] with desired closed-loop time constant $\tau_{c}=12 \mathrm{~min}$ (one-fifth of dominant openloop time constant) is used to design a proportional-integral (PI) controller with proportional gain $K_{c}=$ 77.517 and integral time $\tau_{I}=29.036 \mathrm{~min}$. For comparison purposes, this procedure is repeated for the case $d_{m}=\emptyset$. In this case, the linear model between $T_{i}$ and CV $c=-1.675 C_{A}+1.425 C_{B}+0.0003 T+0.011 T_{i}$, obtained around nominal optimal point, is:

$$
\frac{c(s)}{T_{i}(s)}=\frac{0.0153(40.142 s+1)(14.492 s+1)}{(60 s+1)(13.353 s+1)}
$$

As before, using half-rule and IMC method [Skogestad, 2003], a PI controller with proportional gain $K_{c}=48.495$ and integral time $\tau_{I}=19.858 \mathrm{~min}$ is designed. The closed-loop responses for multiple step changes in disturbances $C_{A i}$ (from 1 to $1.3 \mathrm{~mol} / \mathrm{L}$ at $t=1 \mathrm{~min}$ and from 1.3 to $0.7 \mathrm{~mol} / \mathrm{L}$ at $t=501$ $\min$ ) and $C_{B i}$ (from 0 to $0.3 \mathrm{~mol} / \mathrm{L}$ at $t=251 \mathrm{~min}$ and from 0.3 to $0 \mathrm{~mol} / \mathrm{L}$ at $t=751 \mathrm{~min}$ ) are shown in Figure 2. In these simulations, the measurement errors are considered to be uniformly distributed random sequences with magnitudes $\pm \sum_{i=1}^{n_{y}}\left|H_{y} W_{e}\right|, \pm \sum_{i=1}^{n_{y}}\left|H W_{e}\right|$ and $\pm \sum_{i=1}^{n_{d}}\left|H_{d_{m}} W_{e_{d}}\right|$ for $\tilde{c} . \quad c$ and $\hat{d}_{m}$, respectively. It can be seen that $\tilde{c}$ follows its setpoint $c_{s}$, updated based on measurements of $C_{B i}$, closely with smooth changes in $T_{i}$. The average cost $(J)$ for $d_{m}=\emptyset$ is $0.414[\$ / \mathrm{min}]$, which reduces to $0.395[\$ / \mathrm{min}]$ for $d_{m}=C_{B i}$.

Overall, this case study clearly demonstrates that the proposed method can provide considerably lower loss in comparison with traditional feedback-based SOC policy. We point out that the benefits of incorporating feedforward action in SOC policies strongly depends on the accuracy of disturbance measurement. For example, in comparison with feedback-based SOC policy $\left(d_{m}=\emptyset\right)$, the use of $\tilde{c}$ as CV reduces the average loss computed using nonlinear model by only $8 \%$, if the measurement error of $C_{B_{i}}$ is $0.1 \mathrm{~mol} / \mathrm{L}$.

\subsection{Forced-Circulation Evaporator}

Next, we consider the case of forced-circulation evaporator [Cao, 2005; Kariwala et al., 2008; Newell and Lee, 1989]. The primary objective of this case study is to illustrate the use of price variation as measured disturbance in SOC. The schematic representation for this process is shown in Figure 3 and the variables are listed in Table 2. The operational objective of this process involves minimizing

$$
J=\alpha F_{100}+0.6 F_{200}+1.009\left(F_{2}+F_{3}\right)+0.2 F_{1}-4800 F_{2}
$$

which denotes negative profit. In (27), $\alpha$ denotes the price of steam (virtual disturbance). This process has a number of operational constraints on $X_{2}, P_{2}, P_{100}, F_{200}, F_{1}$, and $F_{3}$; see Kariwala et al. [2008] for 
details.

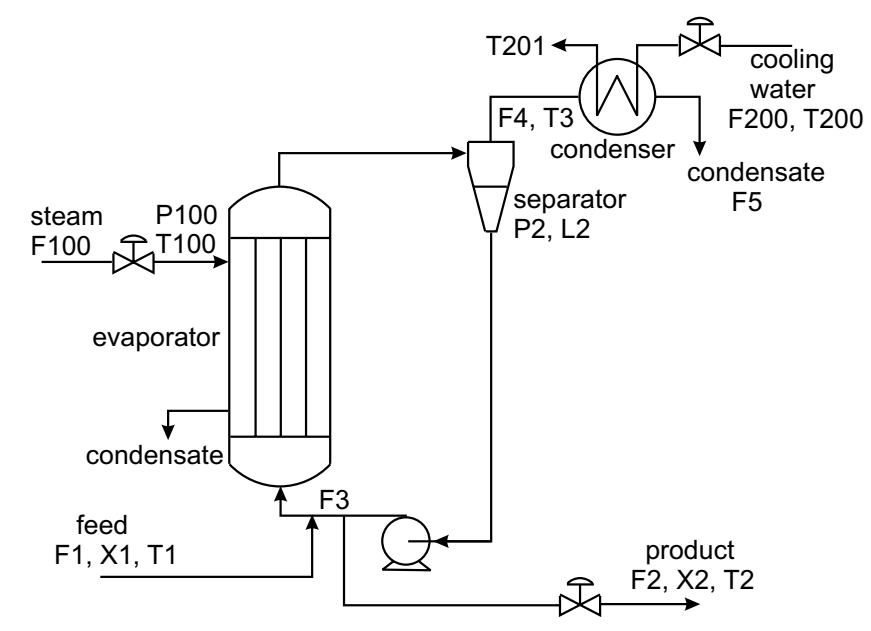

Figure 3: Schematic representation of the Forced Circulation Evaporator

Table 2: Variables of Forced Circulation Evaporator

\begin{tabular}{ll|ll}
\hline Var. & Description & Var. & Description \\
\hline$F_{1}$ & Feed flowrate & $T_{3}$ & Vapor temperature \\
$F_{2}$ & Product flowrate & $L_{2}$ & Separator level \\
$F_{3}$ & Circulating flowrate & $P_{2}$ & Operating pressure \\
$F_{4}$ & Vapor flowrate & $F_{100}$ & Steam flowrate \\
$F_{5}$ & Condensate flowrate & $T_{100}$ & Steam temperature \\
$X_{1}$ & Feed composition & $P_{100}$ & Steam pressure \\
$X_{2}$ & Product composition & $F_{200}$ & Cooling water flowrate \\
$T_{1}$ & Feed temperature & $T_{200}$ & Inlet temperature of cooling water \\
$T_{2}$ & Product temperature & $T_{201}$ & Outlet temperature of cooling water \\
\hline
\end{tabular}

This process has 5 manipulated inputs $\left(F_{1}, F_{2}, P_{100}, F_{3}\right.$, and $\left.F_{200}\right)$ and 3 disturbances $\left(X_{1}, T_{1}\right.$ and $\left.T_{200}\right)$. The case, where $X_{1}=5 \%, T_{1}=40^{\circ} \mathrm{C}, T_{200}=25^{\circ} \mathrm{C}$ and $\alpha=600$, is taken as the nominal operating point. At these conditions, the optimum negative profit of the process is $-582.233[\$ / \mathrm{h}]$. It is noted that the constraints on $X_{2}$ and $P_{100}$ remain active over the entire set of allowable disturbances. In addition, the separator level $\left(L_{2}\right)$ needs to be controlled, which has no steady-state effect. Amongst these 5 manipulated inputs, $P_{100}$ is kept at its upper limit, $F_{2}$ is used to control $X_{2}$ at the lower bound and $F_{3}$ is adopted to maintain the level, $L_{2}$ at its setpoint. Thus, two inputs are available as unconstrained degrees of freedom, for which CVs need to be selected. Without loss of generality, they are taken as $F_{1}$ and $F_{200}$. 
The measurement set is given as

$$
y=\left[\begin{array}{lllllllll}
P_{2} & T_{2} & T_{3} & F_{2} & F_{100} & T_{201} & F_{5} & F_{200} & F_{1}
\end{array}\right]^{\prime}
$$

Note that in comparison with earlier studies [Kariwala et al., 2008], $F_{3}$ is not included in $y$, as the linear model for this measurement results in large plant-model mismatch due to linearization [Kariwala et al., 2008]. The gain and Hessian matrices for this process are available in [Kariwala et al., 2008]. In addition, for $\alpha$

$$
J_{u \alpha}=\left[\begin{array}{ll}
-0.001 & 1.115
\end{array}\right]^{\prime}
$$

The allowable disturbance set corresponds to $\pm 5 \%$ variation in $X_{1}, \pm 20 \%$ variation in $T_{1}$ and $T_{200}$ and $\pm 10 \%$ in $\alpha$ around their nominal values. Based on these variations, we have $W_{d}=\operatorname{diag}(0.25,8,5,60)$. The implementation errors for the pressure and flow measurements are taken to be $\pm 2.5 \%$ and $\pm 2 \%$, respectively, of the nominal operating values. For temperature measurements, implementation error is considered to be $\pm 1^{\circ} C$. Therefore, we have $W_{e}=\operatorname{diag}(1.285,1,1,0.027,0.189,1,0.163,4.355,0.189)$. It is considered that the price $\alpha$ is measured with an accuracy of $0.5 \%$ of its nominal value, which implies $W_{\tilde{e}}=\operatorname{diag}\left(W_{e}, 3\right)$.

Analysis. Let us define $c_{9}$ and $\tilde{c}_{10}$ as the CV candidates consisting of combination of all the measurements in $y$ and the extended measurement set $\tilde{y}^{\prime}=\left[\begin{array}{ll}y^{\prime} & \alpha\end{array}\right]$, respectively. The local average losses incurred with the use of $c_{9}$ and $\tilde{c}_{10}$ as $\mathrm{CVs}$ are $4.306 \$ / \mathrm{h}$ and $0.224 \$ / \mathrm{h}$, respectively. Similarly, the local worst-case loss, when $c_{9}$ and $\tilde{c}_{10}$ are used as $\mathrm{CVs}$, are $167.866 \$ / \mathrm{h}$ and $9.325 \$ / \mathrm{h}$, respectively. This analysis shows that varying the setpoints based on $\alpha$ can significantly reduce the loss.

To obtain simpler control structure, combinations of fewer measurements, which can be used as CVs, are identified using BAB method with local average loss minimization [Kariwala and Cao, 2010]. The BAB method is applied by considering $y$ (without including $\alpha$ in the measurement subset) and $\tilde{y}$ (with inclusion of $\alpha$ in the measurement subset) as the measurement set. For both cases, the variations of least local average losses with the measurement subset size, $n_{y}$ are shown in Figure 4. As $\alpha$ itself, i.e. without other measurements, cannot be used as a $\mathrm{CV}$, combinations of at least 3 measurements need to be selected as CVs, when $\alpha$ is included in the measurement subset. Figure 4 shows that including $\alpha$ in the measurement set reduces the loss by factors of 2-20 for different $n_{y}$.

It can be observed from Figure 4 that when $\alpha$ is included in the measurement subset, the use of combinations of 4 or 5 measurements as CVs yields similar local average loss (0.505 and $0.383 \$ / \mathrm{h}$, respectively) as seen for $\tilde{c}_{10}(0.224 \$ / \mathrm{h})$. These CV candidates offer a good trade off between simpler control structure 


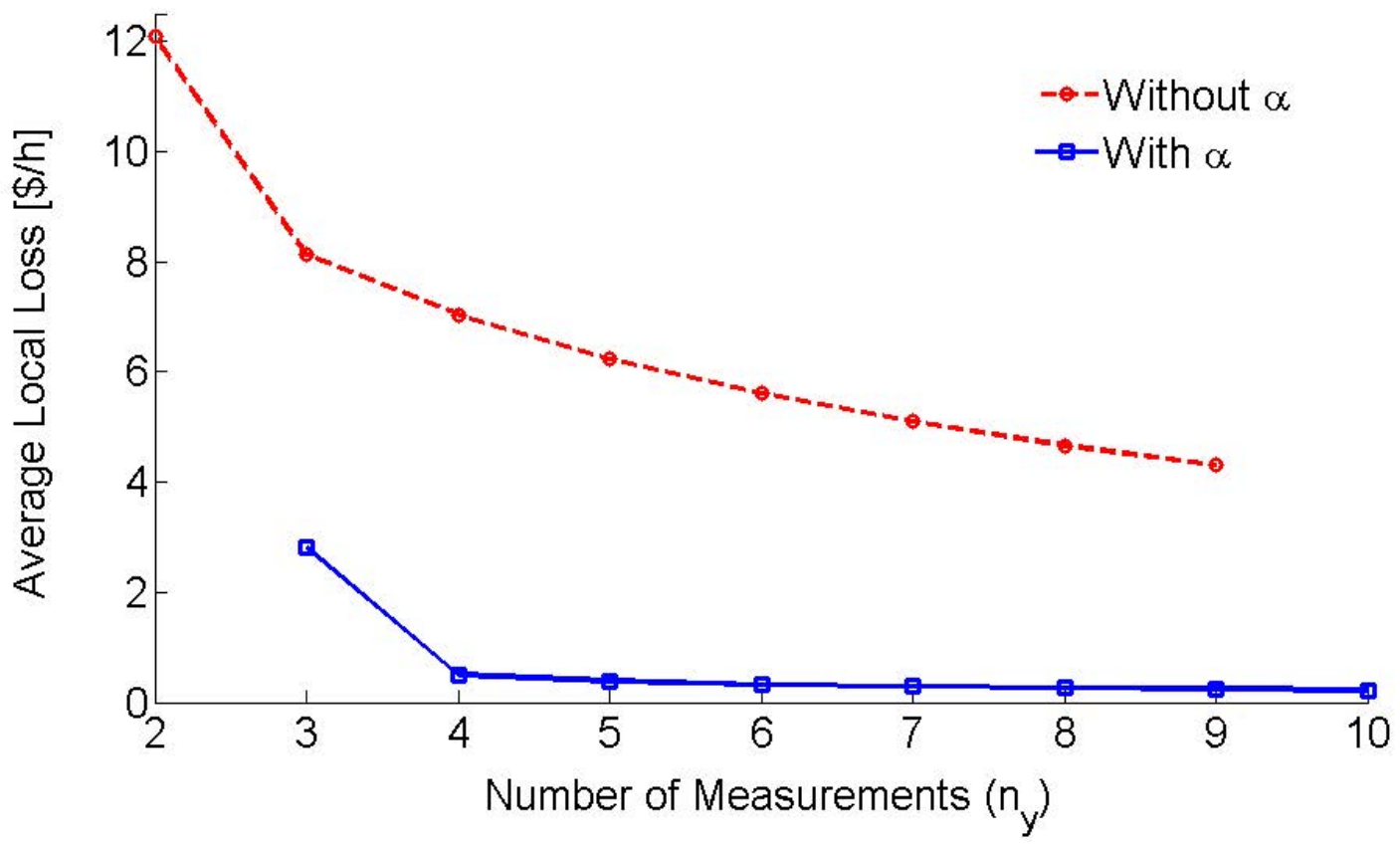

Figure 4: Local average loss for best measurement set with size $n_{y}$

and economic loss, and are given as:

$$
\begin{gathered}
\tilde{c}_{4}=\left[\begin{array}{l}
0.99897 F_{2}+0.027167 F_{100}-0.035538 F_{200}+0.0071889 \alpha \\
0.027401 F_{2}-0.9996 F_{100}+0.0064695 F_{200}+0.0018819 \alpha
\end{array}\right] \\
\tilde{c}_{5}=\left[\begin{array}{c}
0.9991 F_{2}-0.022095 F_{100}+0.000894 F_{5}-0.03539 F_{200}+0.0073234 \alpha \\
-0.021832 F_{2}-0.99973 F_{100}+0.00027481 F_{5}+0.0081462 F_{200}+0.0015411 \alpha
\end{array}\right]
\end{gathered}
$$

Similar to the CSTR case study, nonlinear analysis is conducted to verify the results of local analysis. The resulting average and worst-case losses for the promising candidates are presented in Table 3 . We note that using $\tilde{c}_{4}$ and $\tilde{c}_{5}$ as CVs provides lower losses than the use of $\tilde{c}_{10}$ as CVs. This observation highlights the local optimality of $\tilde{H}$ and approaches to overcome this limitation will be pursued in future. In comparison with $c_{9}$, the use of $\tilde{c}_{5}$ as $\mathrm{CV}$ reduces the average and worst-case losses obtained using nonlinear model by factors of 18 and 8, respectively. Furthermore, the relative average loss evaluated based on the nonlinear model is approximately $0.3 \%$ with the use of $\tilde{c}_{5}$ as $\mathrm{CV}$.

The performance of the proposed method is further evaluated through dynamic simulations using $c_{9}$ and $\tilde{c}_{5}$ as CVs, respectively. For both cases, the first component of CVs (CV1) is cascaded with a pressure controller through $F_{200}$ to maintain $P_{2}$ within its upper and lower constraints, as described in [Cao, 2005], 
Table 3: Local and nonlinear analysis for promising CV alternatives for forced-circulation evaporator

\begin{tabular}{|c|c|c|c|c|c|}
\hline \multirow{2}{*}{ CV } & \multirow{2}{*}{ Measurements } & \multicolumn{2}{|c|}{ Local Analysis } & \multicolumn{2}{|c|}{ Nonlinear Analysis } \\
\hline & & $L_{\text {average }}[\$ / \mathrm{h}]$ & $L_{\text {worst }}[\$ / \mathrm{h}]$ & Average loss $[\$ / \mathrm{h}]$ & Worst-case loss $[\$ / \mathrm{h}]$ \\
\hline$c_{9}$ & $y$ & 4.306 & 167.866 & 36.229 & 180.925 \\
\hline$\tilde{c}_{4}$ & $F_{2}, F_{100}, F_{200}, \alpha$ & 0.505 & 12.032 & 2.267 & 27.349 \\
\hline$\tilde{c}_{5}$ & $F_{2}, F_{100}, F_{200}, F_{5}, \alpha$ & 0.383 & 10.275 & 2.159 & 24.427 \\
\hline$\tilde{c}_{10}$ & $\tilde{y}=\left|\begin{array}{ll}y^{\prime} & \alpha\end{array}\right|$ & 0.224 & 9.325 & 2.399 & 36.885 \\
\hline
\end{tabular}

whilst the second component of CVs (CV2) is paired with the manipulated variable, $F_{1}$. All controller parameters, except those used in the CV2- $F_{1}$ loop, are the same as those reported in [Cao, 2005]. For the CV2- $F_{1}$ loop, a simple PI controller is designed with a unit control gain and unit integral time (1 min). All measurements are assumed to have uniformly distributed measurement noises within the variation ranges specified by $W_{\tilde{e}}$. For simplicity, the process is considered to be operated at the nominally optimal point initially. The following step changes are introduced in $\alpha$ : $\$ 600$ to $\$ 660$ at $t=1$ hours and $\$ 660$ to $\$ 540$ at $t=10$ hours, which lasts until $t=20$ hours, as shown in Figure 5 (a). The profit losses comparing to the true optimal operation resulted due to the use of $\tilde{c}_{5}$ or $c_{9}$ as CVs are shown in 5 (b). The corresponding transient responses of key process variables are shown in Figure 6.
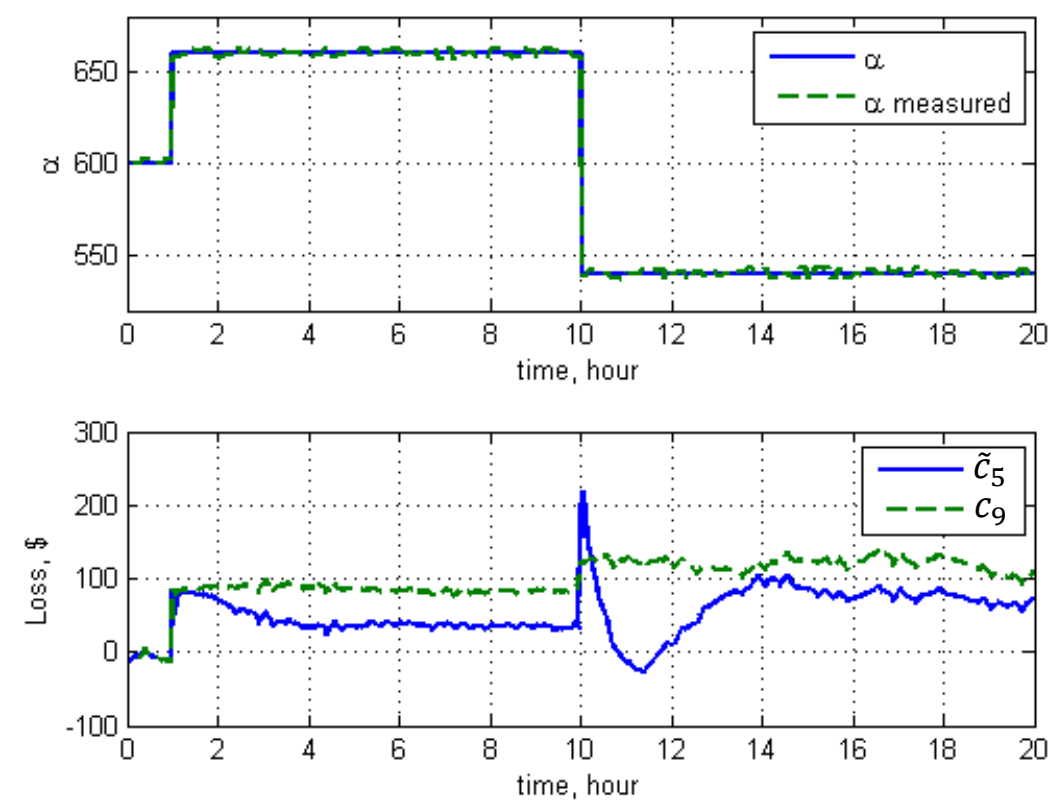

Figure 5: (a) Step changes of $\alpha$ (b) Hourly profit loss comparing to true optimal operation

It can be seen that for the first hour when steam price, $\alpha$ is at nominal value, both $\tilde{c}_{5}$ and $c_{9}$ result 
in almost the same zero loss. However, when $\alpha$ increases or decreases from the nominal value, without feedforward action, $c_{9}$ does not change the operational conditions, as indicated by dashed lines in Figure 6 , resulting in a higher profit loss compared to that obtained by using $\tilde{c}_{5}$ as $\mathrm{CV}$, for which feedforward action is incorporated to adjust operation conditions accordingly, as indicated by the solid lines in Figure 6 .

From Figure 6, it can be seen that when the steam price increases, the CV with feedforward action, $\tilde{c}_{5}$ reduces $F_{1}, F_{2}, F_{3}$ and $P_{2}$, which requires $F_{200}$ to increase, such that the plant operates at a low production mode to keep the operation profitable, whilst, when the steam price decreases, $\tilde{c}_{5}$ is able to adjust process variables so as the plant operates in a high production mode to take the advantage fully. In contrast, the CV without feedforward action, $c_{9}$ cannot detect the steam price changes. Hence, the plant operates continuously in the nominal condition independent from the steam price resulting in high losses.

The total profit for 20 hours of operation is $\$ 13160.95$ and $\$ 12232.23$, when $\tilde{c}_{5}$ and $c_{9}$ are used as CVs, respectively. Based on these results, the use of $\tilde{c}_{5}$ as $\mathrm{CV}$ is recommended for the forced-circulation evaporator.
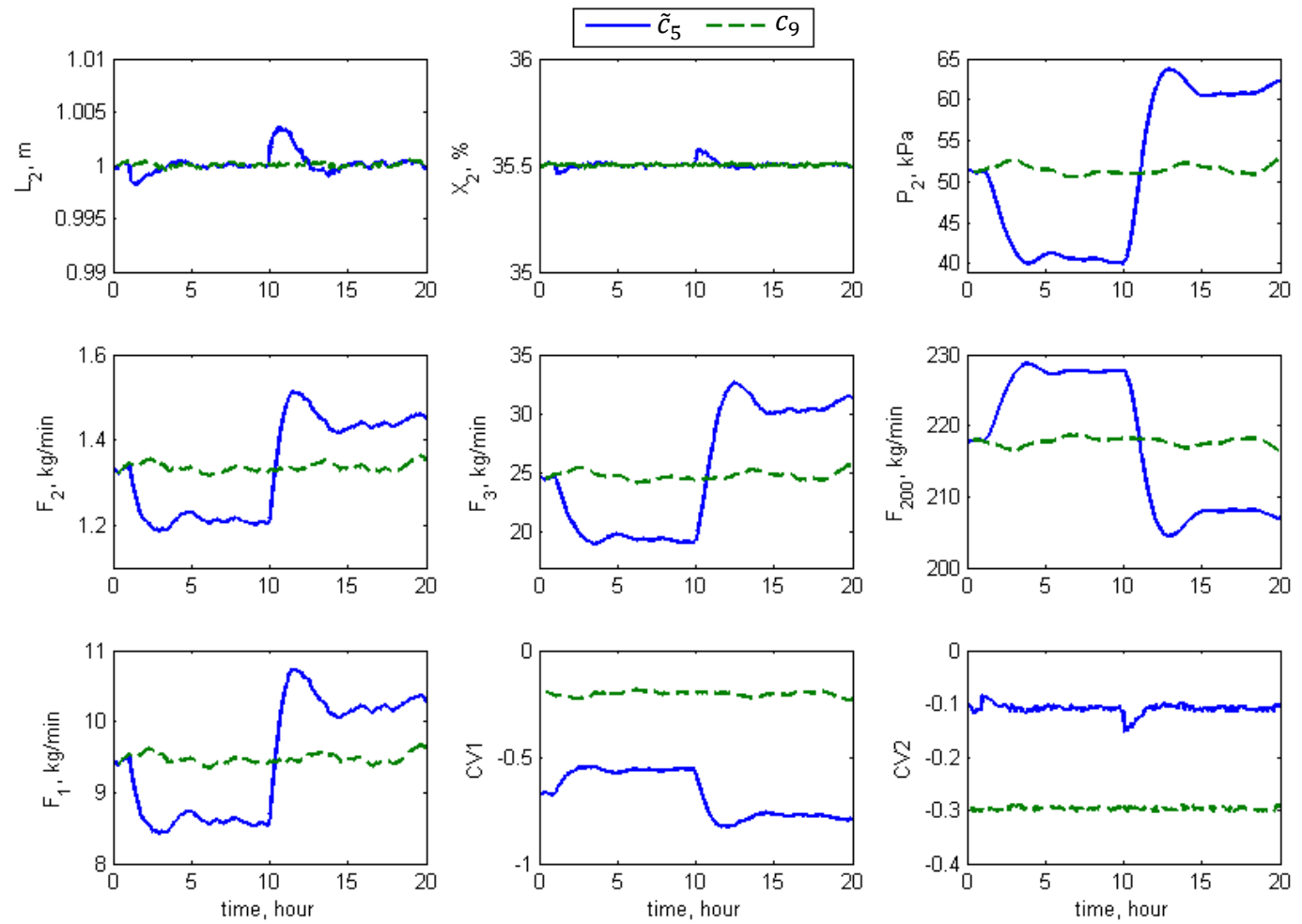

Figure 6: Transient response of major process variables using $c_{5}$ as CVs. 


\section{Conclusions}

We proposed an approach to improve the feedback-based self optimizing control (SOC) by including information of key disturbances into measurement set, whose combinations are used as CVs. The incorporation of the feedforward action is useful to anticipate the effect of measured disturbances and thus lower economic loss is obtained in comparison with the use of CVs synthesized with the feedback-based SOC policies. This work provides broader scope in comparison with the work of Cao and Yang [2004] by extending the notion of measured disturbances to include the physical/process disturbances in addition to the "virtual" disturbances, which only affect the scalar objective function related to optimal operation of process. Case studies of CSTR and forced-circulation evaporator show that incorporation of key disturbance information in SOC policies results in significant reduction of loss. It is pointed out that this reduction in loss requires additional sensors for measuring disturbances and thus selection of dominant disturbances, as done for CSTR case study, is important to minimize the additional cost of instrumentation. Furthermore, unlike individual measurements, measurement combinations lack physical interpretation, which can sometimes limit their adoption as CVs. To overcome this issue, Heldt [2010] and Yelchuru and Skogestad [2012] have suggested imposing structure on $H$ such that only physically similar measurements, e.g. temperature or flow measurements, are combined as CVs, which can be used for incorporating feedforward action in SOC policies as well. Lastly, the available methods for selection of self-optimizing CVs are based on steadystate economics and the selected CVs may suffer from poor dynamic controllability. A possible approach to overcome this drawback is to select CVs based on dynamic models. Some progress to handle process dynamics has been reported in [Dahl-Olsen et al., 2008; Hu et al., 2012], which can form the basis for future research.

\section{References}

Alstad, V., 2005. Studies on selection of controlled variables. Ph.D. thesis, Department of Chemical Engineering, Norwegian University of Science and Technology, Trondheim, Norway.

Alstad, V., Skogestad, S., Hori, E. S., 2009. Optimal measurement combinations as controlled variables. J. Proc. Control 19 (1), 138-148.

Cao, Y., 2005. Direct and indirect gradient control for static optimisation. Internationl Journal of Automation and Computing $2(1), 60-66$.

Cao, Y., Yang, S., 2004. Self-optimizing control for economic variation. Control 2004, Bath, UK. 
Dahl-Olsen, H., Narasimhan, S., Skogestad, S., 2008. Optimal output selection for control of batch processes. In: American Control Conference, 2008. IEEE, pp. 2870-2871.

Economou, C. G., Morari, M., Palsson, B. O., 1986. Internal model control: 5. Extension to nonlinear systems. Ind. Eng. Chem. Process Des. Dev. 25 (2), 403-411.

Halvorsen, I. J., Skogestad, S., Morud, J. C., Alstad, V., 2003. Optimal selection of controlled variables. Ind. Eng. Chem. Res. 42 (14), 3273-3284.

Heldt, S., 2010. Dealing with structural constraints in self-optimizing control engineering. Journal of Process Control 20 (9), 1049-1058.

Hu, W., Mao, J., Xiao, G., Kariwala, V., 2012. Selection of self-optimizing controlled variables for dynamic processes. In: Advanced Control of Chemical Processes. Vol. 8. pp. 774-779.

Jaschke, J., Skogestad, S., 2012. Optimal controlled variables for polynomial systems. J. Proc. Control $22(1), 167-179$.

Kariwala, V., 2007. Optimal measurement combination for local self-optimizing control. Ind. Eng. Chem. Res. 46 (11), 3629-3634.

Kariwala, V., Cao, Y., 2009. Bidirectional branch and bound for controlled variable selection: Part II. Exact local method for self-optimizing control. Comput. Chem. Eng. 33 (8), 1402-1412.

Kariwala, V., Cao, Y., 2010. Bidirectional branch and bound for controlled variable selection: Part III. Local average loss minimization. IEEE Trans. Ind. Informat. 6 (1), 54-61.

Kariwala, V., Cao, Y., Janardhanan, S., 2008. Local self-optimizing control with average loss minimization. Ind. Eng. Chem. Res. 47 (4), 1150-1158.

Newell, R., Lee, P., 1989. Applied Process Control - A Case Study. Prentice Hall, Englewood Cliffs, NJ.

Rangaiah, G. P., Kariwala, V., 2012. Plantwide Control: Recent Developments and Applications. John Wiley \& Sons, Chichester, UK.

Skogestad, S., 2000. Plantwide control: The search for the self-optimizing control structure. J. Proc. Control 10 (5), 487-507.

Skogestad, S., 2003. Simple analytic rules for model reduction and PID controller tuning. J. Proc. Contr. 11, 291-309. 
Skogestad, S., Postlethwaite, I., 1996. Multivariable Feedback Control: Analysis and Design, 1st Edition. John Wiley \& sons, Chichester, UK.

Van de Wal, M., de Jager, B., 2001. A review of methods for input/output selection. Automatica 37 (4), $487-510$.

Yelchuru, R., Skogestad, S., 2012. Convex formulations for optimal selection of controlled variables and measurements using mixed integer quadratic programming. J. Proc. Contr. 22 (6), 995-1007. 